\title{
Coarsening Dynamics of a Nonconserved Field Advected by a Uniform Shear Flow
}

\author{
Alan J. Bray and Andrea Cavagna \\ Department of Physics and Astronomy, The University, Manchester, M13 9PL, UK
}

(October 31, 2018)

\begin{abstract}
We consider the ordering kinetics of a nonconserved scalar field advected by a uniform shear flow. Using the Ohta-Jasnow-Kawasaki approximation, modified to allow for shear-induced anisotropy, we calculate the asymptotic time dependence of the characteristic length scales, $L_{\|}$and $L_{\perp}$, that describe the growth of order parallel and perpendicular to the mean domain orientation. In space dimension $d=3$ we find $L_{\|} \sim \gamma t^{3 / 2}, L_{\perp} \sim t^{1 / 2}$, where $\gamma$ is the shear rate, while for $d=2$ we find $L_{\|} \sim \gamma^{1 / 2} t(\ln t)^{1 / 4}, L_{\perp} \sim \gamma^{-1 / 2}(\ln t)^{-1 / 4}$. Our predictions for $d=2$ can be tested by experiments on twisted nematic liquid crystals.
\end{abstract}

The coarsening dynamics of systems quenched from a disordered phase into a two-phase region are by now reasonably well understood [i]. Domains of the ordered phases form rapidly, and then coarsen with time $t$, i.e. there is a characteristic length scale ('domain size'), $L(t)$, which grows with time, typically as a power law. Furthermore, there is good evidence for a form of dynamical scaling in which the domain morphology is statistically scale-invariant when all lengths are scaled by $L(t)$ [1].

In recent years attention has been directed at systems subjected to external driving, e.g. an imposed shear [2. ent coarsening rates in directions parallel and perpendicular to the flow. This has been observed in experiments [3].

An important open question for such driven systems is whether the coarsening continues indefinitely (for an infinite system), as in the case of no driving, or whether the driving force arrests the coarsening, leading to a steady state. For the case of a sheared phase-separating binary fluid, it has been argued, on the basis of the stability of a single drop of one fluid immersed in another, that the domain scale will eventually saturate at a maximum length scale, $L_{\max }$, determined by the shear rate: $L_{\max } \sim \sigma / \gamma \eta$, where $\sigma$ and $\eta$ are the surface tension and viscosity respectively [2]. In the multi-domain context, it has been argued [5] that a steady state would be achieved through the shear-induced stretching and breaking of domains. However, the experimental evidence for a steady state is not completely clear-cut. In particular, as emphasized by Cates et al. [6], saturation of the domain length occurs naturally in a finite-size system when the domain length becomes of the same order as the system size.

A second important question concerns the nature of the growth laws, and the nature of scaling - if it exists - in this anisotropic system. Naively, one might expect two characteristic length scales, $L_{\|}$and $L_{\perp}$, measuring correlations parallel and perpendicular to the flow. In dimension $d=2$, for example, it would be natural to conjecture that the coarsening follows conventional scaling when lengths along and perpendicular to the flow are scaled by these characteristic lengths. We will show, however, that the actual scaling is subtly different.
To address these issues it would be helpful to have an exactly soluble model. The large- $n$ limit of the $n$-vector model has been solved for a conserved order-parameter field, but without hydrodynamics (i.e. the order parameter is simply advected by the shear flow) [7]. Length scales $L_{\|}$and $L_{\perp}$, growing as $\gamma\left(t^{5} / \ln t\right)^{1 / 4}$ and $(t / \ln t)^{1 / 4}$ respectively, describe correlations along and perpendicular to the flow. Scaling is not strictly satisfied (there is instead a form of 'multiscaling'), but this is presumably an artifact of the large- $n$ limit, as in the zero-shear case [8]. There is no saturation at late times in this model. However, for the large- $n$ model there are no domains, so the concept of stretching and breaking loses its meaning. What is needed is an analytically tractable model for a scalar order parameter. For a nonconserved field, the Ohta-Jasnow-Kawasaki (OJK) approximation [9] fulfills this requirement: In the absence of shear, it captures the essential features of the coarsening process [1].

In this Letter we present the results of applying the OJK approach to a nonconserved scalar field advected by a uniform shear flow. The equation of motion for the order parameter is

$$
\partial_{t} \phi+\nabla \cdot(\mathbf{u} \phi)=\nabla^{2} \phi-V^{\prime}(\phi),
$$

where $V(\phi)$ is a symmetric double well potential, and $\mathbf{u}=\gamma y \hat{\mathbf{x}}$ is the velocity of the imposed shear flow, with the flow in the $x$-direction. Two aspects of real binary fluids are neglected in this model: the order parameter is not conserved, and it is simply advected by the shear rather than being coupled to the fluid velocity through the Navier-Stokes equation. This approach is, however, a very instructive first step which yields important insights concerning the main questions raised earlier, namely the nature the asymptotics and of dynamical scaling, in the physically correct context of a scalar field. Furthermore, in $d=2$ this model describes the coarsening dynamics of a twisted nematic liquid crystal, in which disclination lines, separating domains of opposite twist, relax viscously, driven by their line tension 10]. Under shear, this system will furnish an experimental test of our predictions. It should be noted that our analysis leads to very different behavior in $d=2$ and $d=3$ : This is one of the main results of the present work. 
The result of the OJK analysis is that, for space dimension $d=3, L_{\|} \sim \gamma t^{3 / 2}$ and $L_{\perp} \sim t^{1 / 2}$, i.e. the coarsening rate parallel to the flow is enhanced by a factor $\gamma t$ relative to the unsheared system, while the growth of $L_{\perp}$ is unchanged (the same features describe the large- $n$ result [7]). Hence there is no saturation of the coarsening. For $d=3$, furthermore, conventional scaling holds. For $d=2$, however, very different results are obtained: $L_{\|} \sim \gamma^{1 / 2} t(\ln t)^{1 / 4}$, and $L_{\perp} \sim \gamma^{-1 / 2}(\ln t)^{-1 / 4}$. These results imply $L_{\|} L_{\perp} \sim t$ for $d=2$, i.e. the product of the two length scales, or 'scale area', is independent of the shear rate and has the same form as for the unsheared system, where $L_{\|}=L_{\perp} \sim t^{1 / 2}$. We will show that this result can be understood by a topological argument. An important subtlety in $d=2$ is that $L_{\|}$and $L_{\perp}$ have to be defined as characteristic scales parallel and perpendicular to the mean domain orientation, instead of the flow direction. This distinction is not important for $d=3$, but crucial for $d=2$. Only with the new definition is dynamical scaling recovered. For $d=2$, furthermore, $L_{\perp}$ decreases with time asymptotically. Our approach breaks down, however, when $L_{\perp}$ becomes comparable with the width, $\xi$, of the interfaces between domains, which occurs after a time of order $\exp \left(\right.$ const $\left./ \gamma^{2} \xi^{4}\right)$, when we expect the domains to break, as observed in simulations with conserved dynamics in $d=2[5]$.

The OJK approach starts from the Allen-Cahn equation [11] relating the normal component of the interface velocity, $v_{n}$, to the local curvature of the interface, $K=\nabla \cdot \mathbf{n}$, where $\mathbf{n}$ is the normal to the interface,

$$
v_{n}=-\nabla \cdot \mathbf{n}+\mathbf{u} \cdot \mathbf{n}
$$

where the final term is the drift due to the shear. The derivation of this equation from (11) follows the same route as the zero-shear case [1]. The next step is to introduce a smooth auxiliary field $m(\mathbf{x}, t)$ whose zeroes coincide with those of $\phi$. In a frame comoving with the interface one has $d m / d t=0=\partial_{t} m+v_{n}|\nabla m|$. Combining this with (2), and using

$$
\mathbf{n}=\nabla m /|\nabla m|
$$

yields the following equation for $m$,

$$
\partial_{t} m+\mathbf{u} \cdot \nabla m=\nabla^{2} m-\sum_{a, b=1}^{d} n_{a} n_{b} \partial_{a} \partial_{b} m .
$$

So far this is exact. Equation (4) is highly non-linear, however, due to the implicit dependence of $\mathbf{n}$ on $m$ through (3). The OJK approximation involves linearizing the $m$ equation by replacing the product $n_{a} n_{b}$ by its spatial average,

$$
D_{a b}(t)=\left\langle n_{a}(\mathbf{x}, t) n_{b}(\mathbf{x}, t)\right\rangle,
$$

leading to the following equations for $m$ and $D$ :

$$
\begin{aligned}
\partial_{t} m+\gamma y \partial_{x} m & =\nabla^{2} m-D_{a b}(t) \partial_{a} \partial_{b} m \\
D_{a b} & =\left\langle\frac{\partial_{a} m \partial_{b} m}{(\nabla m)^{2}}\right\rangle .
\end{aligned}
$$

In the absence of shear the coarsening is isotropic, and the matrix $D$ has the simple form $D_{a b}=\delta_{a b} / d$, independent of $t$. The resulting diffusion equation for $m$ is readily solved, leading to a $\sqrt{t}$ coarsening. For $\gamma \neq 0$, the coarsening is anisotropic: $D_{a b}$ is both non-diagonal and timedependent. From the definition (5) of $D_{a b}$, though, the sum-rule, $\operatorname{Tr} D=1$, is trivially valid. From the symmetry of (6) under the combined transformations $x \rightarrow-x$, $y \rightarrow-y$ at fixed $z$, and under the separate transformation $z \rightarrow-z$ at fixed $x, y$, we see that $D_{a b}$ has a block diagonal form, with $D_{x y}=D_{y x}$ the only non-zero off-diagonal elements.

In Fourier space, (6) reads

$$
\begin{aligned}
\frac{\partial m(\mathbf{k}, t)}{\partial t}-\gamma k_{x} \frac{\partial m(\mathbf{k}, t)}{\partial k_{y}} & =-\sum_{a b} \Omega_{a b}(t) k_{a} k_{b} m(\mathbf{k}, t) \\
\Omega_{a b}(t) & =\delta_{a b}-D_{a b}(t) .
\end{aligned}
$$

This is readily solved by the change of variables $\mathbf{q}=A \mathbf{k}$, $\tau=t$, and $\mu(\mathbf{q}, \tau)=m(\mathbf{k}, t)$, where $A$ has elements

$$
A_{a b}=\delta_{a b}+\gamma t \delta_{a 2} \delta_{b 1}
$$

In the new variables, the left-hand side of (\$) becomes $\partial_{\tau} \mu(\mathbf{q}, \tau)$. Integrating the equation, and transforming back to the original variables, gives

$$
m(\mathbf{k}, t)=m(\tilde{\mathbf{k}}(t), 0) \exp \left(-\frac{1}{4} \sum_{a b} k_{a} M_{a b}(t) k_{b}\right),
$$

where $\tilde{\mathbf{k}}(t)=\left(k_{x}, k_{y}+\gamma k_{x} t, k_{z}\right)$ and the matrix $M$ is given by

$$
\begin{aligned}
M(t) & =A^{T}(t) R(t) A(t) \\
R(t) & =4 \int_{0}^{t} d t^{\prime}\left[A^{T}\left(t^{\prime}\right)\right]^{-1} \Omega\left(t^{\prime}\right)\left[A\left(t^{\prime}\right)\right]^{-1} .
\end{aligned}
$$

Equations (11-13) determine the function $m(\mathbf{k}, t)$ completely if the matrix $D_{a b}(t)$ is known. However, $D$ is itself determined from the distribution for $m$, via (7), so we have to solve these equations self-consistently. We take the initial condition, $m(\mathbf{k}, 0)$ to be a gaussian random variable with correlator $\left\langle m(\mathbf{k}, 0) m\left(\mathbf{k}^{\prime}, 0\right)\right\rangle=\Delta \delta\left(\mathbf{k}+\mathbf{k}^{\prime}\right)$. Then the real-space correlation function of $m, G(\mathbf{r}, t)=$ $\langle m(\mathbf{x}+\mathbf{r}, t) m(\mathbf{x}, t)\rangle$ is obtained from (11) as

$$
G(\mathbf{r}, t)=G(0, t) \exp \left(-\frac{1}{2} \sum_{a, b} r_{a}\left(M^{-1}\right)_{a b} r_{b}\right)
$$

where the precise expression for $G(0, t)$ is not relevant in what follows. All the information concerning domain growth in this system is contained in the matrix $M_{a b}(t)$. 
To obtain a closed set of equations we have to express the elements of the matrix $D$ in terms of the elements of $M$. To to this we first rewrite (7) using an integral representation for the denominator:

$$
D_{a b}=\frac{1}{2} \int_{0}^{\infty} d u\left\langle\partial_{a} m \partial_{b} m \exp \left(-\frac{u}{2}(\nabla m)^{2}\right)\right\rangle .
$$

Since $m$ is a Gaussian field, the required average can be computed using the probability distribution $P(\mathbf{v})$ of the vector $\mathbf{v}=\nabla m$. This distribution is determined by the correlator $\left\langle v_{a} v_{b}\right\rangle=-\left.\partial_{a} \partial_{b}\langle m(\mathbf{r}, t) m(\mathbf{0}, t)\rangle\right|_{\mathbf{r}=\mathbf{0}}=$ $\left\langle m^{2}\right\rangle\left(M^{-1}\right)_{a b}$, from which one infers that

$$
P(\mathbf{v}) \propto \exp \left(-\frac{1}{2\left\langle m^{2}\right\rangle} \sum_{a, b} v_{a} M_{a b} v_{b}\right) .
$$

Carrying out the average in (15) gives

$$
\begin{aligned}
D_{a b} & =\frac{1}{2} \int_{0}^{\infty} d u\left(\frac{\operatorname{det} M}{\operatorname{det} N}\right)^{1 / 2}\left(N^{-1}\right)_{a b} \\
N_{a b} & =M_{a b}+u \delta_{a b} .
\end{aligned}
$$

We now proceed to outline the solution of this closed set of equations. Full details will be given elsewhere [12]. We are interested in the large- $t$ asymptotics. This limit simplifies the analysis which is still, however, quite subtle. The results are very different in three and two dimensions, so we discuss these cases separately.

We begin with some general remarks on the expected form of $D_{a b}$. The effect of the shear is to produce elongated domain structures aligned, at late times, at a small angle ( $\sim 1 / \gamma t$ - see below) to the flow direction. As a result, the component $n_{1}$ of the normal to the interface is very small almost everywhere at late times, implying $D_{11} \rightarrow 0$ for $t \rightarrow \infty$. The sum rule, $\operatorname{Tr} D=1$, is therefore exhausted by the remaining diagonal components of $D$ for large $t$. In particular, for $d=2$ we have $D_{22} \rightarrow 1$ for $t \rightarrow \infty$, and $\Omega_{11} \rightarrow 0$ in (8). This case requires special care. For $d=3$, on the other hand, $D_{22}+D_{33} \rightarrow 1$, and it turns out that both $D_{22}$ and $D_{33}$ approach non-zero limits. This case is, therefore, simpler to analyse.

The case $\mathbf{d}=\mathbf{3}$. With the assumption that $D_{11} \rightarrow 0$ and $D_{12} \sim 1 / t$, while $D_{22}$ and $D_{33}$ remain non-zero for $t \rightarrow \infty$, the asymptotics of the matrix elements $M_{a b}$ are readily obtained from (9), (10), (12) and (13):

$$
\begin{aligned}
& M_{11}=\frac{4}{3} \gamma^{2} t^{3}\left(1-D_{22}^{\infty}\right) \\
& M_{12}=2 \gamma t^{2}\left(1-D_{22}^{\infty}\right) \\
& M_{22}=4 t\left(1-D_{22}^{\infty}\right) \\
& M_{33}=4 t D_{22}^{\infty},
\end{aligned}
$$

where $D_{22}^{\infty}$ is the large- $t$ limit of $D_{22}$, while $M_{13}=M_{23}=$ 0 by symmetry.
Using these limiting forms, the integrals (17) can be evaluated asymptotically. After some algebra one finds

$$
D_{22}^{\infty}=\left(1+\frac{1}{2}\left(\frac{1-D_{22}^{\infty}}{D_{22}^{\infty}}\right)^{1 / 2}\right)^{-1}
$$

while $D_{33}^{\infty}=1-D_{22}^{\infty}$ and $D_{13}=D_{23}=0$. Equation (20) has the non-trivial solution $D_{22}^{\infty}=4 / 5$, implying $D_{33}^{\infty}=1 / 5$ and demonstrating the self-consistency of the initial ansatz. Finally we find $D_{11} \rightarrow 3 \ln (\gamma t) /(\gamma t)^{2}$ and $D_{12} \rightarrow-6 /(5 \gamma t)$, consistent with our initial assumption.

The characteristic length scales in the system are given, from (14), by the square roots of the eigenvalues of the matrix $M$. Using (19), with $D_{22}^{\infty}=4 / 5$, we find

$$
L_{\|}=\frac{2}{\sqrt{15}} \gamma t^{3 / 2}, \quad L_{\perp}=\frac{1}{\sqrt{5}} t^{1 / 2}, \quad L_{3}=\frac{4}{\sqrt{5}} t^{1 / 2},
$$

for $t \rightarrow \infty$. The corresponding eigenvectors are

$$
\mathbf{e}_{\|}=\left(\begin{array}{c}
1 \\
\frac{3}{2 \gamma t} \\
0
\end{array}\right), \quad \mathbf{e}_{\perp}=\left(\begin{array}{c}
-\frac{3}{2 \gamma t} \\
1 \\
0
\end{array}\right), \quad \mathbf{e}_{3}=\left(\begin{array}{l}
0 \\
0 \\
1
\end{array}\right),
$$

implying that the principal axes in the $x y$ plane are rotated, relative to the $x$ and $y$ axes, through an angle $3 / 2 \gamma t$, which we can interpret as the angle between the average orientation of the domain structure and the flow direction.

The three length scales are all distinct, though $L_{\perp}$ and $L_{3}$ grow in the same way, and coarsening continues indefinitely - the system does not approach a stationary state. The matrix elements $M_{a b}$ grow in the way expected if a naive form of scaling holds: $M_{11}=L_{\|}^{2}, M_{12} \sim L_{\|} L_{\perp}$, $M_{22} \sim L_{\perp}^{2}$, and $M_{33}=L_{3}^{2}$. This means that dynamical scaling holds, and the scaling variables can be taken to be $x / L_{\|}, y / L_{\perp}$, and $z / L_{3}$. The same simple structure does not, however, hold in $d=2$.

The case $\mathbf{d}=\mathbf{2}$. For $d=2$ the self-consistency problem is more tricky, because the quantity $1-D_{22}(t)=$ $D_{11}(t)$ tends to zero as $t \rightarrow \infty$. For $d=2$ the integrals (17) can be evaluated exactly. Making the assumptions, to be verified subsequently, that asymptotically $M_{11} \gg M_{12} \gg M_{22}$, that $\operatorname{Tr} M \gg \sqrt{\operatorname{det} M} \gg M_{22}$, and that $D_{12} \sim 1 / t$, one can derive the following selfconsistent equation for $D_{11}(t)$ :

$$
D_{11}(t)=\frac{1}{\gamma t^{2}}\left(\frac{\int_{0}^{t} d t^{\prime} t^{\prime 2} D_{11}\left(t^{\prime}\right)}{\int_{0}^{t} d t^{\prime} D_{11}\left(t^{\prime}\right)}\right)^{1 / 2},
$$

with asymptotic solution

$$
D_{11}(t)=\frac{1}{2 \gamma t \sqrt{\ln \gamma t}}
$$

Using this result, the asymptotic results for the matrix elements, and the determinant, of the correlation matrix $M$ are obtained as 


$$
\begin{aligned}
M_{11}(t) & =4 \gamma t^{2} \sqrt{\ln \gamma t} \\
M_{12}(t) & =4 t \sqrt{\ln \gamma t} \\
M_{22}(t) & =(4 / \gamma) \sqrt{\ln \gamma t} \\
\operatorname{det} M(t) & =4 t^{2},
\end{aligned}
$$

while $D_{12}=-1 / \gamma t$. These results confirm, a posteriori, the assumptions made in their derivation, i.e. the solution is self-consistent. The asymptotic results for the $M_{a b}$ seem to imply $\operatorname{det} M=0$, in contradiction to (25). To obtain (25) one has to keep subdominant contributions to the $M_{a b}$. [Note that $\operatorname{det} M=\operatorname{det} R$ from (12)].

The characteristic length scales are given, as before, by the square roots of the eigenvalues of $M$. The eigenvalues of any $2 \times 2$ matrix can be expressed as $\lambda_{ \pm}=$ $\left[\operatorname{Tr} M \pm \sqrt{(\operatorname{Tr} M)^{2}-4 \operatorname{det} M}\right] / 2$. Using $\operatorname{Tr} M \gg \sqrt{\operatorname{det} M}$ we obtain $\lambda_{+}=\operatorname{Tr} M$ and $\lambda_{-}=\operatorname{det} M /(\operatorname{Tr} M)$, whence

$$
L_{\|}=2 \sqrt{\gamma} t(\ln \gamma t)^{1 / 4}, \quad L_{\perp}=\frac{1}{\sqrt{\gamma}(\ln \gamma t)^{1 / 4}} .
$$

The corresponding eigenvectors are

$$
\mathbf{e}_{\|}=\left(\begin{array}{c}
1 \\
\frac{1}{\gamma t}
\end{array}\right), \quad \mathbf{e}_{\perp}=\left(\begin{array}{c}
-\frac{1}{\gamma t} \\
1
\end{array}\right)
$$

giving a tilt angle $1 / \gamma t$ between the domain orientation and the flow direction.

The scale area in two dimensions is $L_{\|} L_{\perp}=2 t$, independent of $\gamma$. This is the same result as the zero-shear case, where $D_{a b}=\delta_{a b} / d$ implies $M_{a b}=2 t \delta_{a b}$ for $d=2$, i.e. $L(t)=\sqrt{2 t}$. This result is special to $d=2$ and can be understood as follows. For an isolated domain, the rate of change of the area enclosed by the domain boundary is $d A / d t=\oint d l v_{n}=\oint d l(\mathbf{u}-\nabla) \cdot \mathbf{n}$ from (2). The second term is a topological invariant, equal to $-2 \pi$ from the Gauss-Bonnet Theorem, while the first term is equal to $\int_{A} d^{2} x \nabla \cdot \mathbf{u}$, which vanishes for any divergence-free shear flow. While the $\gamma$-independence of $d A / d t$ has been proved only for closed loops of domain wall, we expect a similar result to hold for the scale area, i.e. $d\left(L_{\|} L_{\perp}\right) / d t=$ const. It is very encouraging that the OJK approximation captures this feature of the $d=2$ coarsening. There is no equivalent result in $d=3$ because the surface integral $\int d S \nabla \cdot \mathbf{n}$ is no longer a topological invariant.

The scaling is nontrivial in $d=2$ because, although $M_{11}=L_{\|}^{2}$, consistent with naive scaling, the corresponding results $M_{12} \sim L_{\|} L_{\perp}, M_{22} \sim L_{\perp}^{2}$, found in $d=3$ no longer hold in $d=2$. This is associated with the fact that the leading-order contribution to $\operatorname{det} M$ vanishes. The consequence is that scaling only holds when referred to the unique scaling axes (27), which are themselves time-dependent.

The most interesting and suggestive feature of the $d=2$ result is that $L_{\perp}$ tends to zero as $t \rightarrow \infty$. Since our treatment is based on the 'thin wall' limit, in which domain walls are treated as having zero width, it will break down when $L_{\perp}$ becomes comparable with the width, $\xi$, of the walls, at which point we conjecture that domains will break, possibly arresting the coarsening. This can be tested by experiments on twisted nematic liquid crystals. In $d=3$, the present work provides strong evidence that, at least for the nonconserved scalar field considered here, the coarsening state proceeds indefinitely. In this respect it is interesting that, in their $d=3$ simulations (including both conservation of the order parameter and hydrodynamics), Cates et al. [6] found no evidence for a steady state structure emerging that is independent of the system size, i.e. observed steady states could be attributed to finite size effects.

We thank Peter Sollich for a useful discussion. This work was supported by EPSRC (UK) under grant GR/L97698.

[1] A. J. Bray, Adv. Phys. 43, 357 (1994), and references therein.

[2] A. Onuki, J. Phys.: Condens. Matter 9, 6119 (1997), and references therein.

[3] C. K. Chan, F. Perrot, and D. Beysens, Phys. Rev. A 43, 1826 (1991); A. H. Krall, J. V. Sengers, and K. Hamano, Phys. Rev. Lett. 69, 1963 (1992); T. Hashimoto, K. Matsuzaka, E. Moses, and A. Onuki, Phys. Rev. Lett. 74, 126 (1995); J. Läuger, C. Laubner, and W. Gronski, Phys. Rev. Lett. 75, 3576 (1995).

[4] D. H. Rothman, Phys. Rev. Lett. 65, 3305 (1990); P. Padilla and S. Toxvaerd, J. Chem. Phys. 106, 2342 (1997); A. J. Wagner and J. M. Yeomans, Phys. Rev. E 59, 4366 (1999).

[5] T. Ohta, H. Nozaki, and M. Doi, Phys. Lett. A 145, 304 (1990); J. Chem. Phys. 93, 2664 (1991).

[6] M. E. Cates, V. M. Kendon, P. Bladon, and J.-C. Desplat, Faraday Discuss. 112, 1 (1999).

[7] N. P. Rapapa and A. J. Bray, Phys. Rev. Lett. 83, 3856 (1999); F. Corberi, G. Gonnella, and A. Lamura, Phys. Rev. Lett. 81, 3852 (1998).

[8] A. Coniglio and M. Zannetti, Europhys. Lett. 10, 575 (1989); A. J. Bray and K. Humayun, Phys. Rev. Lett. 68, 1559 (1992).

[9] T. Ohta, D. Jasnow, and K. Kawasaki, Phys. Rev. Lett. 49, 1223 (1982).

[10] H. Orihara and Y. Ishibashi, J. Phys. Soc. Jpn. 55, 2151 (1986).

[11] S. M. Allen and J. W. Cahn, Acta Metall. 27, 1085 (1979).

[12] A. Cavagna and A. J. Bray, in preparation. 